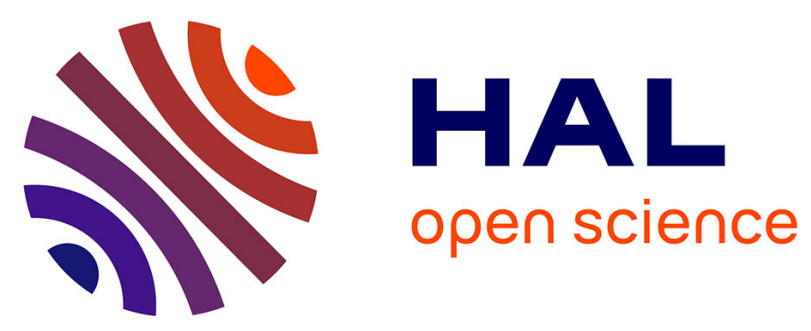

\title{
Contact and Mobility Simulation for Mechanical Assemblies based on Skin Model Shapes
} Benjamin Schleich, Nabil Anwer, Luc Mathieu, Sandro Wartzack

\section{To cite this version:}

Benjamin Schleich, Nabil Anwer, Luc Mathieu, Sandro Wartzack. Contact and Mobility Simulation for Mechanical Assemblies based on Skin Model Shapes. Journal of Computing and Information Science in Engineering, 2015, pp.7. 10.1115/1.4029051 . hal-01094269

\section{HAL Id: hal-01094269 https://hal.science/hal-01094269}

Submitted on 12 Dec 2014

HAL is a multi-disciplinary open access archive for the deposit and dissemination of scientific research documents, whether they are published or not. The documents may come from teaching and research institutions in France or abroad, or from public or private research centers.
L'archive ouverte pluridisciplinaire HAL, est destinée au dépôt et à la diffusion de documents scientifiques de niveau recherche, publiés ou non, émanant des établissements d'enseignement et de recherche français ou étrangers, des laboratoires publics ou privés. 


\title{
Skin Model Based Simulation of Contact and Mobility for Mechanical Assemblies
}

\author{
Benjamin Schleich \\ Nabil Anwer, Luc Mathieu \\ Chair of Engineering Design Automated Production Research Laboratory \\ Department of Mechanical Engineering Ecole Normale Superieure de Cachan \\ Friedrich-Alexander-University Erlangen-Nürnberg 94230 Cachan, France \\ 91058 Erlangen, Germany \\ Email: schleich@mfk.fau.de
}

\author{
Sandro Wartzack \\ Chair of Engineering Design \\ Department of Mechanical Engineering \\ Friedrich-Alexander-University Erlangen-Nürnberg \\ 91058 Erlangen, Germany \\ Email: schleich@mfk.fau.de
}

\begin{abstract}
Assembly modelling as one of the most important steps in the product development activity relies more and more on the extensive use of CAD systems. The modelling of geometric interfaces between the components of the assembly is of central importance in mechanical simulation of assemblies.
\end{abstract}

Over the past decades, many researches have devoted their efforts to establish theories and systems covering assembly modelling. Although the product form or shape have been extensively investigated considering the nominal CAD geometry, inevitable limitations can be reported. Computer Aided Tolerancing systems provide simulation tools for modelling the effects of tolerances on the assembly situation but still lack of form deviation considerations. The skin model concept which stemmed from the theoretical foundations of Geometrical Product Specification and Verification (GPS) has been developed to enrich the nominal geometry considering realistic physical shapes. However, the digital representation of the skin model has been investigated only recently.

This paper presents a novel approach for a skin model based simulation of contact and mobility for assemblies. Three important issues are addressed: the geometric modelling of the contact, the contact quality evaluation, and the motion analysis. The main contribution to computer aided tolerancing can be found in the analysis of the effects of geometric form deviations on the assembly and motion behaviour of solid mechanics, which comprises models for the assembly simulation, for the contact quality evaluation, and for the motion analysis. A case study is presented to illustrate the proposed approaches.

\author{
Nomenclature \\ CAD Computer-Aided Design \\ CAT Computer-Aided Tolerancing \\ GPS Geometric Product Specification and Verification
}

\section{Introduction}

Assembly modelling as one of the most important steps in the product development process relies more and more on the extensive use of CAD systems. Digital mock-ups enable virtual assembly and testing without building physical artefacts while reducing time and costs in product development and increasing product quality. The modelling of geometric interfaces between the components of the assembly is of central importance in mechanical simulations of assemblies.

Over the past decades, many researches have devoted their efforts to establish theories and systems covering assembly modelling. Although the product form or shape have been extensively investigated considering the nominal CAD geometry, inevitable limitations can be reported. Computer Aided Tolerancing (CAT) systems provide simulation tools for modelling the effects of tolerances on the assembly function. Integration of manufacturing simulations and physical modelling into tolerance analysis lead to more accurate methods but still lack of form deviation considerations. The skin model concept which stemmed from the theoretical foundations of Geometrical Product Specification (GPS) has been developed to enrich the nominal geometry considering realistic physical shapes. The skin model considers geometric deviations that are expected, predicted or already observed in real manufacturing and assembly processes. How- 
ever, the digital representation of the skin model has been investigated only recently.

This paper presents a novel approach for a skin model based simulation of contact and mobility for assemblies. Three important issues are addressed: the geometric modelling of the contact, the contact quality evaluation, and the motion analysis. It is structured as follows. In the following section, GeoSpelling as a coherent and complete language for Geometric Product Specification and Verification, the Skin Model, and the concept of Skin Model Shapes are briefly introduced. Thereafter, existing approaches for the assembly simulation of non-ideal workpiece representatives are reviewed. Following this, a framework for the assembly and mobility simulation of Skin Model Shapes is proposed. This framework is then applied to a case study of a slider mechanism. Finally, a conclusion is given.

\section{GeoSpelling, the Skin Model, and the Concept of Skin Model Shapes}

The industrial need for a coherent and complete language for geometric product specification and verification has led to the development of GeoSpelling and its adoption in the standards for GPS (ISO 17450-1:2011) [1-4]. In GeoSpelling, a geometric specification is defined as a condition on a characteristic defined on geometric features, which are created from the Skin Model employing different operations. In this regard, the Skin Model is a model of the physical interface between the workpiece and its environment and, in contrast to the Nominal Model, comprises deviations brought in by manufacturing and assembly processes. Since an infinite description is required in order to consider all kinds of geometric deviations, there is no possibility for identification and simulation of the Skin Model. Therefore, the concept of Skin Model Shapes has been developed, which provides a finite descriptiveness of the Skin Model employing either discrete or parametric geometry representation schemes [5-7]. In this contribution, a focus is set on the discrete geometry representation by point clouds, since it is easy to handle and can serve as a connection between design, manufacturing, and inspection. This is because point clouds can be generated by tessellation employing CAD systems during design, result from manufacturing process simulation software tools, and are commonly obtained from tactile or optical measurement systems during inspection. The difference between the Nominal Model, the Skin Model, and the concept of Skin Model Shapes can be seen from Figure 1 .

\section{Assembly Simulation in Computer Aided Tolerancing}

Assembly modelling and simulation has been the topic of many relevant research works in the field of computer aided tolerancing during the last decades. Whereas simple models for the 2D assembly stack-up have been the focus of early research works [8], the representation and simulation of $3 \mathrm{D}$ assembly problems considering geometric part deviations in computer aided tolerancing tools remains an un-

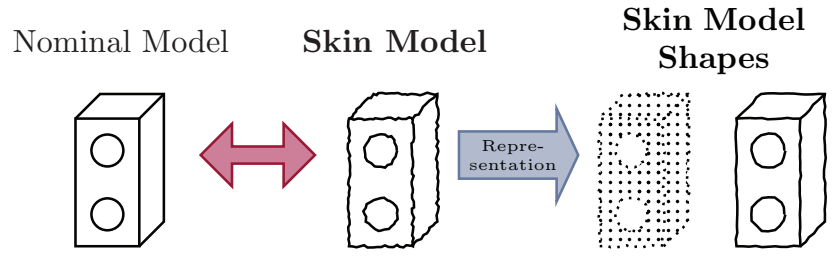

Fig. 1. The Difference between the Nominal Model, the Skin Model, and the Concept of Skin Model Shapes

solved problem [9]. Though, many research works have been presented, which differ in their model assumptions and with regard to their applicability. For example, Li and RoY [10] propose a relative positioning scheme, which is used to determine the positions of variant polyhedral parts in 3D assemblies. Furthermore, a numerical procedure for the assembly simulation among variational features has been proposed in $[11,12]$. Both approaches can be used to model and simulate assemblies with variant parts, but lack of form deviation considerations.

In contrast to that, SAMPER et al. [13] take into account form deviations of planar features in the computation of assemblies. The approach is based on the modal description of form defects and part of a modal tolerancing approach, which also comprises the modal parametrization of form defects [14]. Thus, the simulation results depend on the approximation of form deviations by eigenmodes. This limitation is overcome in the approaches by STOLL and WITTMANN $[15,16]$, which are based on surface registration techniques. They adopt the iterative closest point (ICP) algorithm [17] and integrate collision detection approaches [18] for the contact evaluation. However, these approaches can only handle discrete geometry representation schemes, which offer a closed surface representation, such as surface meshes, but are not capable of simulating the assembly behaviour of variant parts based on their point cloud representation. Since point clouds are commonly obtained by assembly and measurement applications, their consideration in CAT tools is highly desirable to enable the connection between design, manufacturing, and inspection, which is the motivation for the presented assembly and mobility simulation approach.

\section{Assembly and Mobility Simulation of Skin Model Shapes}

The proposed framework for the assembly and mobility simulation of Skin Model Shapes can be divided into four stages, namely a Pre-Processing stage, an Assembly Modelling step, the Mobility Modelling, and Post-Processing activities (see Figure 2), where the assembly and mobility modelling can be regarded as the processing of Skin Model Shapes.

During the Pre-Processing, Skin Model Shapes are generated employing either mathematical approaches for the modelling of geometric deviations in early design stages (prediction stage), or by applying methods based on the statistical shape analysis in later stages (observation stage) $[5-7,19,20]$. These Skin Model Shapes are then prepared 


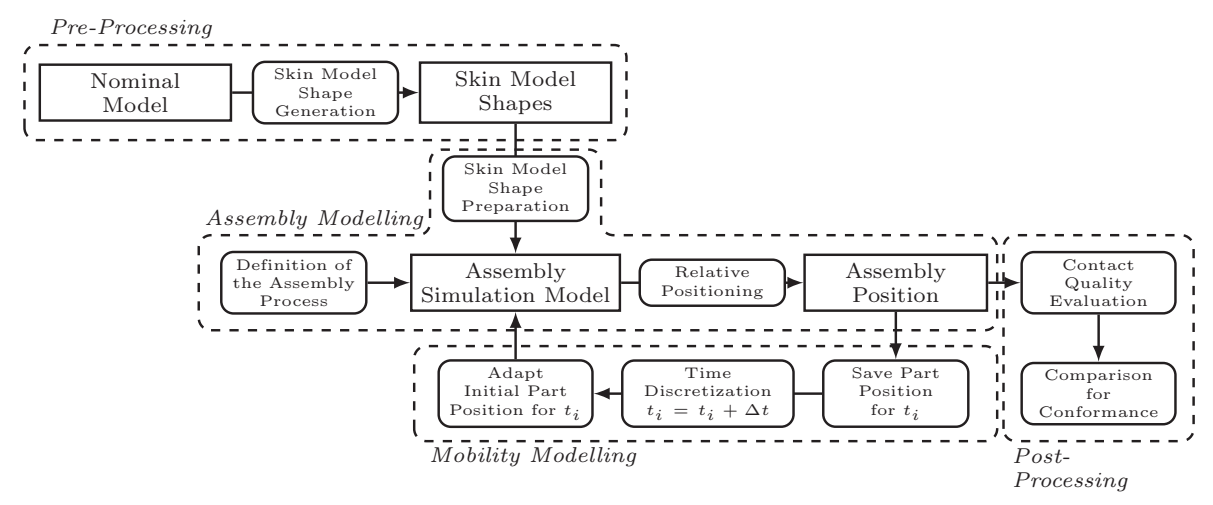

Fig. 2. Simulation Framework

for the Assembly Modelling. In this regard, GeoSpelling operations, such as partition, extraction, and association are applied to obtain the part features, which are relevant for the assembly. Parallel to this, the assembly process and the assembly sequence have to be defined. Both the assembly process definition and the Skin Model Shapes preparation lead to the assembly simulation model, which is then solved employing an approach for the relative positioning. As a result, the relative assembly positions are obtained. The Mobility Modelling is realized as a repetition of single assembly steps, where a time discretization is performed, i. e. the initial part position for every time step $t_{i}+\Delta t$ is adapted based on the previous time step $t_{i}$. Finally, the Post-Processing activities comprise the contact quality evaluation based on the assembly positions, which serves as a criterion for the result interpretation, and the comparison for conformance with assembly requirements. Based thereon, critical workpiece deviations can be identified and geometric part specifications can be derived. The Assembly Modelling and the Mobility Modelling are highlighted in the subsequent subsections.

\subsection{Model for the Assembly Simulation}

The main idea behind the relative positioning of two Skin Model Shapes as proposed in this contribution is based on the registration of their point clouds, where the point cloud of the first part $X$ ( $N$ points) stays unaffected and the point cloud of the second part $Y$ ( $M$ points) is moved relatively to the first part. The adjusted position of $Y$ is obtained by minimizing an objective function $f(\cdot)$, which is a function of both point clouds and the rigid body transformation of the second part $\alpha(Y)$ :

$$
\min f(\alpha(Y) ; X, Y) \text {. }
$$

In order to express the rigid body transformation $\alpha$, we linearize it as $\alpha(Y) \approx m(Y)=Y+v(Y)$ according to PotTMAnN et al. [21], where:

$$
v(Y)=t+r \times Y .
$$

Since this represents an affine transformation, which is not necessarily a rigid body transformation, the resulting veloc- ity vector field is projected to the corresponding helical motion after the optimization (see [21] for further detail). With $t, r \in \mathbb{R}^{3}$, this leads to a minimization problem of dimension six:

$$
\min _{t, r} f(m(Y) ; X, Y)
$$

For the purpose of considering assembly processes with invariant and constrained degrees of freedom, constraints for certain components of $t$ and $r$ are added. The framework for the relative positioning, which is based on [15], can be seen from Figure 3.

In the following, several objective functions for the assembly simulation are presented and discussed, which are illustrated for two planes (point cloud representation) in Figure 4 .

- Convex Hull Volume: The idea behind this objective function is to minimize the volume between the features of both parts. For this purpose, the volume of their joint convex hull is computed and minimized. In this regard, the objective functions yields to:

$$
f_{\text {Hull }}(\cdot)=C V(X \cup m(Y)),
$$

where $C V(\cdot)$ returns the convex hull volume.

- Euclidean Distance: Similarly to the ICP algorithm, the sum of the squared Euclidean point-to-point distances serves as the objective function. However, in the proposed approach, these point-to-point distances are not computed for all point combinations between $X$ and $Y$, but for every point of the moving part $Y$, the correspond-

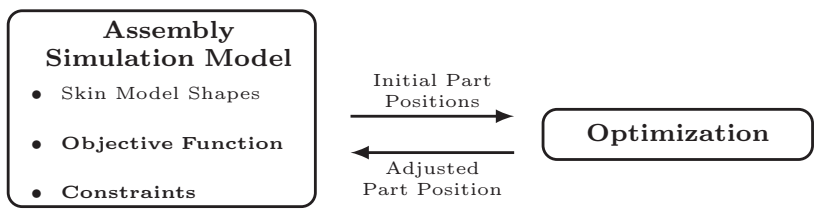

Fig. 3. The Relative Positioning Framework following [15] 


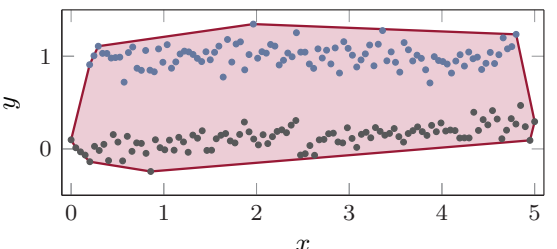

(a) $f_{\text {Hull }}(\cdot)$

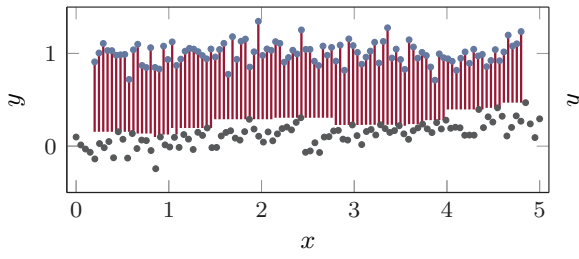

(c) $f_{\mathrm{W}-\mathrm{Assembly}}(\cdot)$

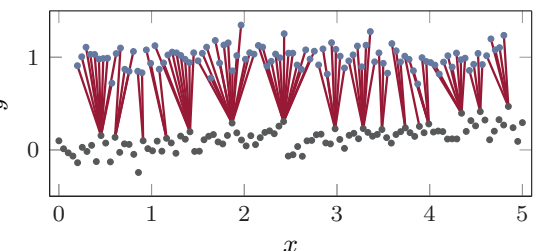

(b) $f_{\text {Euclid }}(\cdot)$

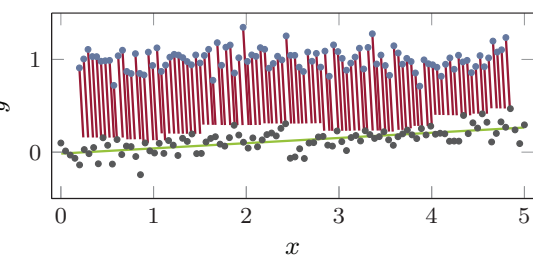

(d) $f_{\mathrm{W}-\mathrm{Normal}}(\cdot)$

Fig. 4. The Objective Functions for the Minimization

ing point in the fixed part $X$ is determined. For this purpose, the nearest neighbour for every point of the moving part $y_{i}$ based on the Euclidean distance is computed:

$$
x_{y_{i}}=\underset{j}{\arg \min }\left\|y_{i}-x_{j}\right\| .
$$

Based thereon, the sum of Euclidean distances between all points of $Y$ and their correspondences in $X$ is minimized, i. e. the objective function yields to:

$$
f_{\text {Euclid }}(\cdot)=\sum_{i=1}^{M} d_{E}\left(m\left(y_{i}\right), x_{y_{i}}\right)^{2},
$$

where $d_{E}\left(m\left(y_{i}\right), x_{y_{i}}\right)$ returns the Euclidean distance between the adjusted point $m\left(y_{i}\right)$ and its nearest neighbour $x_{y_{i}}$, i. e. $d_{E}\left(m\left(y_{i}\right), x_{y_{i}}\right)=\left\|m\left(y_{i}\right)-x_{y_{i}}\right\|$.

- Weighted unsigned distance: Since assembly processes are usually performed along a certain predominant direction, this direction is used to determine the weighted unsigned distance between the point in $Y$ and their correspondences:

$$
f_{\mathrm{W}}(\cdot)=\sum_{i=1}^{M} d_{W}\left(m\left(y_{i}\right), x_{y_{i}}, w\right),
$$

where $d_{W}\left(m\left(y_{i}\right), x_{y_{i}}, w\right)$ returns the weighted distance between the adjusted point $m\left(y_{i}\right)$ and $x_{y_{i}}$ : $d_{W}\left(m\left(y_{i}\right), x_{y_{i}}, w\right)=\left|\left(m\left(y_{i}\right)-x_{y_{i}}\right) \cdot w^{\prime}\right|$. In order to define the weight-vector $w=\left[w_{1} w_{2} w_{3}\right]^{T}$, two approaches are proposed.

- Assembly direction: The weights are defined following the assembly direction, which is the main direction for the assembly step in the global coordinate system. For example, if the feature assembly is performed in $z$-direction, the weights yield to $w=\left[\begin{array}{lll}0 & 0 & 1\end{array}\right]^{T}$. We denote the objective function using this weighted unsigned distance $f_{\mathrm{W} \text {-Assembly }}(\cdot)$.
- Fixed part normal: The weights correspond to the surface normal of the mating plane of the fixed part. The normal vector of the mating plane is evaluated applying the principal component analysis on the fixed mating plane, where the normal can be found as the third principal component. The objective function is denoted by $f_{\mathrm{W}-\mathrm{Normal}}(\cdot)$.

- Weighted signed distance: In contrast to the weighted unsigned distance, the weighted signed distance can be seen as an indicator whether or not the parts collide. However, since a minimization of the signed distance would lead to a highly negative value of the objective function, its absolute value is used:

$$
f_{\mathrm{WS}}(\cdot)=\sum_{i=1}^{M}\left|d_{W S}\left(m\left(y_{i}\right), x_{y_{i}}, w\right)\right|
$$

Similarly to the weighted unsigned distance, $d_{W S}\left(m\left(y_{i}\right), x_{y_{i}}, w\right)$ returns the weighted signed distance between the adjusted point $m\left(y_{i}\right)$ and $x_{y_{i}}$ : $w^{T} \cdot\left(m\left(y_{i}\right)-x_{y_{i}}\right)=w_{1}\left(m\left(y_{i}\right)^{x}-x_{j}^{x}\right)+w_{2}\left(m\left(y_{i}\right)^{y}-\right.$ $\left.x_{j}^{y}\right)+w_{3}\left(m\left(y_{i}\right)^{z}-x_{j}^{z}\right)$. In order to define the weightvector $w=\left[\begin{array}{lll}w_{1} & w_{2} & w_{3}\end{array}\right]^{T}$, we use the approaches explained in the previous item.

Furthermore, the weighted signed distance function can be added as a constraint for the minimization of the weighted unsigned distance functions [22]. In this regard, the minimization problem (3) yields to:

$$
\begin{gathered}
\min _{t, r} f_{\mathrm{W}}(\cdot) \\
\text { subject to } d_{W S}\left(m\left(y_{i}\right), x_{y_{i}},-w\right) \leq 0 \quad \forall y_{i} \in Y
\end{gathered}
$$

In this context, the same weight vector $w$ is used for the objective function and the constraints.

Figure 5 shows the results of the relative positioning for two simple point clouds and different objective functions 


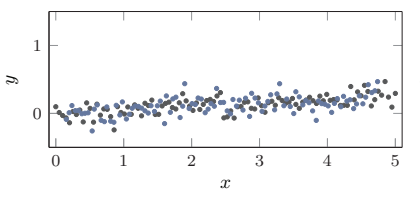

(a) $f_{\text {Hull }}(\cdot)$

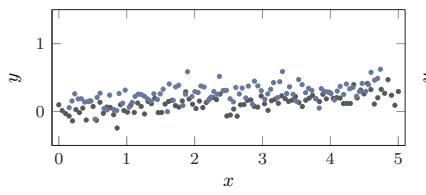

(c) $f_{\text {W-Assembly }}(\cdot)$

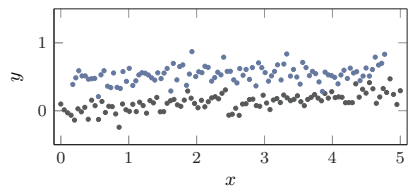

(e) $f_{\mathrm{W}-\text { Assembly }}(\cdot)($ con.)

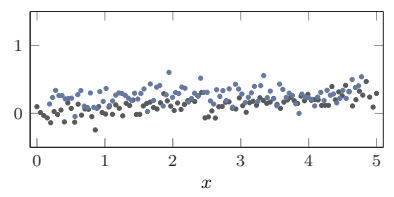

(b) $f_{\text {Euclid }}(\cdot)$

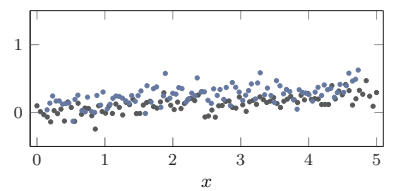

(d) $f_{\mathrm{W}-\mathrm{Normal}}(\cdot)$

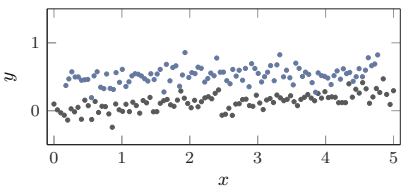

(f) $f_{\text {W-Normal }}(\cdot)$ (con.)
Fig. 5. Results of the Assembly Simulation for two exemplary Point Clouds

with and without constraints. It can be seen, that the adding of constraints based on signed weighted distances (Fig. 5 (e) and (f)) leads to less intersecting part positions, whereas the minimization of the convex hull volume (Fig. 5 (a)) results in a "tight" assembly. This issue is addressed in the following subsection.

\subsection{Contact Quality Evaluation}

Since the point cloud representation of Skin Model Shapes does not provide a closed workpiece surface, it is not possible to precisely determine and simulate the contact between parts in an assembly. Thus, for comparing different approaches and objective functions for the relative positioning, a contact quality evaluation has to be performed. For this purpose, the signed weighted assembly distance $d_{W S}\left(m\left(y_{i}\right), x_{y_{i}}, w\right)$ can be used, since it enables a straightforward interpretation: any negative value of the signed weighted assembly-direction distance is a sign of interpenetration, whereas large values indicate a floating position. In order to evaluate the contact quality of the resulting assembly, the distribution of the point-to-point weighted signed distance are analysed.

Figure 6 shows the histograms of the assembly weighted signed distance $d_{W S}$ for the different objective functions. These distances are obtained by determining the assembly weighted signed distance as defined in equation (8) using the assembly direction as weighting vector $w$ after the assembly simulation for the point clouds from Figures 5. It can be seen, that the convex hull volume minimization leads by trend to an interpenetration of the assembly parts (comparably large amount of negative distances), whereas the constrained weighted distance functions tend to a floating contact (comparably large amount of positive distances). Therefore, the adding of constraints, particularly by using the signed assembly-direction weighted distance, leads to more realistic results for rigid part assemblies.

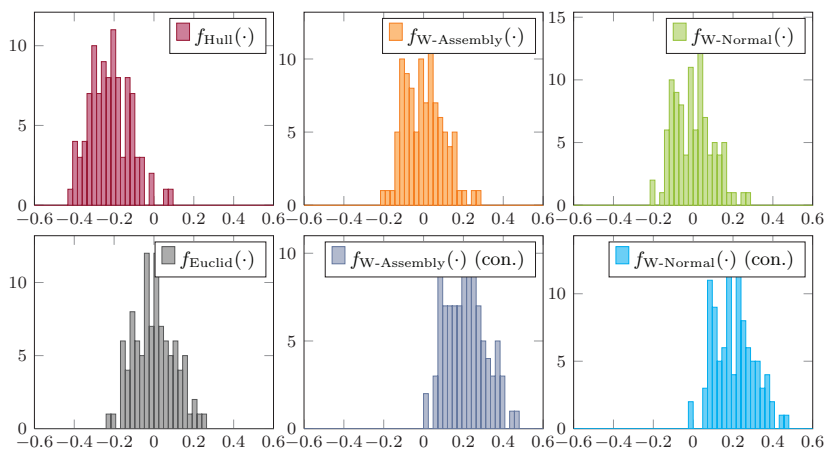

Fig. 6. Histogram of the Signed Weighted Distance for different Objective Functions
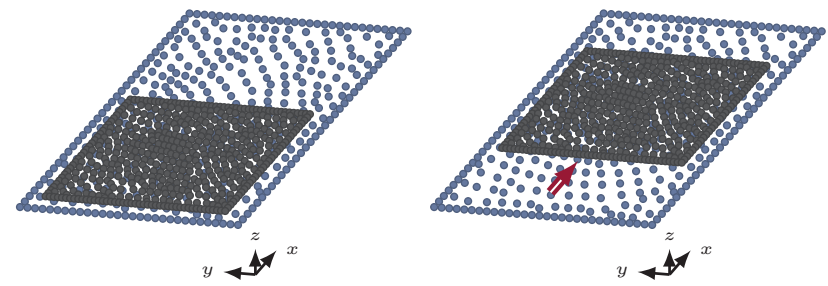

(a) Initial Position in $x$-direction for (b) Initial Position in $x$-direction for $t_{0}$

$t_{i}$

Fig. 7. Adaption of the Initial Part Position for discrete Time Steps

\subsection{Simulation of Mobility}

The mobility simulation is treated as a sequence of assembly simulations for different initial part positions. Thereby, the motion behaviour of moving mechanism can be simulated considering geometric part deviations. In order to perform this, the motion time is discretized to a finite number of time steps $t_{i}$, where the distance between these time steps $\Delta t$ depends on the discretization level. For each of these time steps, the initial position of the moving part is set according to the nominal pre-assembly position for the corresponding time step, which can be seen from Figure 7, where the initial part position is altered in $x$-direction according to the current time step $t_{i}$. Based on the adapted part position, the assembly simulation is performed. Finally, the resulting part position is used to obtain the trajectory of the moving part in the postprocessing stage.

\section{Application and Results}

The approaches for the assembly and mobility simulation are applied to a case study of a slider mechanism as illustrated in Figure 8. The gray block is translated along the $x$-direction guided by the blue clip. In this regard, the key characteristic is the deflection of the block's trajectory in $x$-, $y$-, and $z$-direction compared to the trajectory of the nominal parts.

\subsection{Pre-Processing and Assembly Modelling}

The simulation of assembly and mobility for the slider mechanism starts with the generation of Skin Model Shapes for both parts. For this purpose, a random field approach 


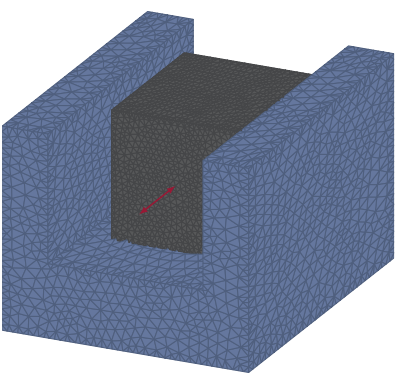

(a) Mesh Representation

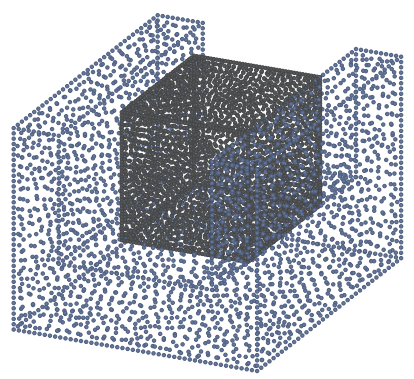

(b) Point cloud Representation
Fig. 8. Slider Mechanism
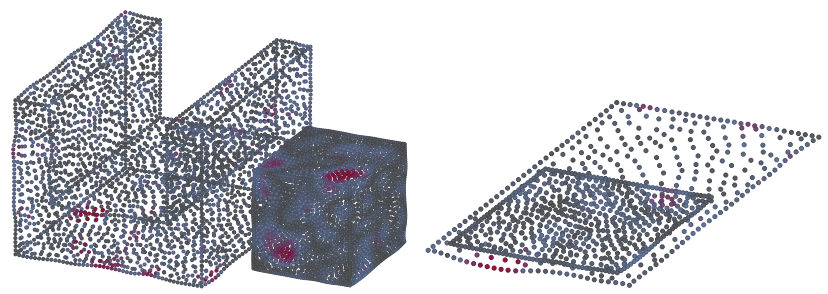

(a) Skin Model Shapes of the Block (b) Partitioning of the Skin Model and the Clip Shapes

Fig. 9. Partitioning of the Skin Model Shapes for the Assembly Modelling

is selected, which is based on the deviation of each part's points along their vertex normals according to spatially correlated random variables $[5,6]$. Thus, spatially correlated Gaussian random values are generated and all points $x_{i} \in X$ and $y_{j} \in Y$ of the tessellated nominal model are deviated in the direction of their local normals by these values. For this purpose, we chose an isotropic, zero-mean Gaussian random field $(\mu=0.0)$ with a correlation length of $l_{\rho}=25.0 \mu m$ and a homogeneous standard deviation of $\sigma=1.0 \mu \mathrm{m}$, which results in flatness deviations from 2.5 to $6.5 \mu \mathrm{m}$. Figure 9 (a) illustrates resulting Skin Model Shapes for both the block and the clip, where the colors indicate the geometric deviations with respect to the nominal model.

The Skin Model Shapes are then prepared for the assembly modelling by the application of GeoSpelling operations, i.e. the relevant workpiece features are extracted, which leads to two planes represented by point clouds, as can be seen from Figure 9 (b). Furthermore, the assembly process is defined, as a straight plane-to-plane assembly in $z$ direction. For the resulting assembly simulation model, the relative positioning is performed employing different objective functions, as previously highlighted.

\subsection{Mobility Modelling}

For the simulation of the kinematic behaviour, the motion space of the block in $x$-direction is discretized in 11 steps. For each of these steps, the initial pre-assembly position is adapted and the relative positioning is performed. As a result, the assembly position for each of the motion steps are obtained. Based thereon, the trajectory of the block is determined. Figure 10 (a) shows the trajectories of 10 Skin

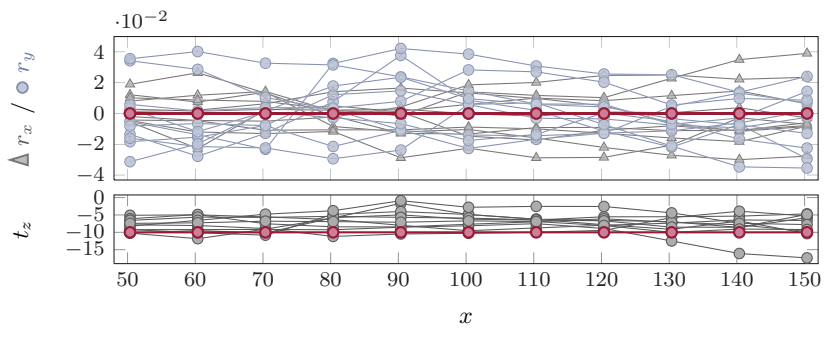

Fig. 10. Trajectories of Skin Model Shapes of the Slider Mechanism

Table 1. Mean Computing Time for the Relative Positioning $(M=$ 532)

\begin{tabular}{r|r|r|r}
$f_{\text {Hull }}(\cdot)$ & $f_{\text {Euclid }}(\cdot)$ & $f_{\text {W-Assembly }}(\cdot)$ & $f_{\text {W-Assembly }}(\cdot)($ con.) \\
\hline $11.6349 \mathrm{~s}$ & $4.2990 \mathrm{~s}$ & $20.0446 \mathrm{~s}$ & $2.8481 \mathrm{~s}$
\end{tabular}

Model Shapes for the translation employing the constrained assembly-direction weighted distance as the objective function $\left(f_{\mathrm{W}-\text { Assembly }}(\cdot)\right)$. It can be seen, that the geometric deviations influence the motion of the block in two manners: firstly, the form deviations of both planes lead to a translation of the block in $z$-direction $\left(t_{z}\right)$ compared to the nominal trajectory (red), and secondly, they lead to rotations of the block around the $x$ - and $y$-axis $\left(r_{x}, r_{y}\right)$ because of tipping. By specifying geometric form tolerances, these effects can be reduced. Thus, the presented approach can support tolerancing decisions during the design of mechanism.

\subsection{Discussion}

The presented approach for the assembly and mobility simulation of Skin Model Shapes represented by point clouds enables the prediction of the effects of geometric deviations on the assembly positions and the kinematic behaviour. It focuses on a geometric consideration of rigid part assemblies and employs numerical optimization approaches for the contact estimation. Several objective functions for the optimization have been presented, where it can be found, that the constrained assembly-direction weighted distance leads to promising results. Furthermore, the required computation time is admissible compared to the other functions as can be seen from Table 1. However, an unsolved issue of the approach is the neglect of physical phenomena, such as friction, gravity, and part compliance. Despite this, by adjusting the parameters of the objective functions, such as the weight vector $w$, this lack can be partly compensated.

\section{Conclusion and Outlook}

In this paper, a framework for the simulation of the assembly and kinematic behaviour of non-ideal workpieces represented by point clouds is proposed. This approach for the assembly and mobility simulation of Skin Model Shapes is capable of predicting the effects of geometric part deviations on the functional requirements in tolerance analysis. It can be found, that the results of the optimization-based simulation of assembly positions, which is depicted as rel- 
ative positioning, highly depend on the employed objective function. The main benefit of this framework can be seen in enabling a coherent and complete tolerancing process based on GeoSpelling as an univocal language for the Geometric Product Specification and Verification. However, future research efforts will focus on the generalization of this framework to more complex assembly processes, the consideration of the assembly sequence in order to simulate multi-step assemblies, and on regarding physical phenomena, such as friction and gravity. Moreover, the validation of the proposed approaches with measurement results will be conducted.

\section{References}

[1] Ballu, A., Mathieu, L., and Dantan, J.-Y., 2003. "Global view of geometrical specifications". In Geometric Product Specification and Verification: Integration of Functionality, P. Bourdet and L. Mathieu, eds. Springer Netherlands, pp. 13-24.

[2] Mathieu, L., and Ballu, A., 2007. "A model for a coherent and complete tolerancing process". In Models for Computer Aided Tolerancing in Design and Manufacturing, J. Davidson, ed. Springer Netherlands, pp. 3544.

[3] Dantan, J.-Y., Ballu, A., and Mathieu, L., 2008. "Geometrical product specifications - model for product life cycle". Computer-Aided Design, 40(4), pp. $493-$ 501.

[4] Anwer, N., Ballu, A., and Mathieu, L., 2013. “The skin model, a comprehensive geometric model for engineering design”. CIRP Annals - Manufacturing Technology, 62(1), pp. 143 - 146.

[5] Schleich, B., Walter, M., Wartzack, S., Anwer, N., and Mathieu, L., 2012. "A comprehensive framework for skin model simulation". In Proceedings of the ASME 2012 11th Biennial Conference on Engineering Systems Design and Analysis, Nantes, ASME, ed., no. ESDA2012-82204, pp. 567-576.

[6] Schleich, B., Anwer, N., Mathieu, L., and Wartzack, S., 2014. "Skin model shapes: A new paradigm shift for geometric variations modelling in mechanical engineering”. Computer-Aided Design, 50(0), pp. 1 - 15.

[7] Anwer, N., Schleich, B., Mathieu, L., and Wartzack, S., 2014. "From solid modelling to skin model shapes: Shifting paradigms in computer-aided tolerancing”. CIRP Annals - Manufacturing Technology, 63(1), pp. $137-140$.

[8] Chase, K., and Parkinson, A., 1991. "A survey of research in the application of tolerance analysis to the design of mechanical assemblies". Research in Engineering Design, 3(1), pp. 23-37.

[9] Hong, Y., and Chang, T., 2002. "A comprehensive review of tolerancing research". International Journal of Production Research, 40(11), pp. 2425-2459.

[10] Li, B., and Roy, U., 2001. "Relative positioning of toleranced polyhedral parts in an assembly". IIE Transactions, 33(4), pp. 323-336.

[11] Franciosa, P., Gerbino, S., and Patalano, S., 2010.
Product Lifecycle Management: Geometric Variations. ISTE. John Wiley \& Sons, Inc., ch. Modeling and Simulation of Assembly Constraints in Tolerance Analysis of Rigid Part Assemblies, pp. 209-229.

[12] Franciosa, P., Gerbino, S., and Patalano, S., 2010. "Variational modeling and assembly constraints in tolerance analysis of rigid part assemblies: planar and cylindrical features". The International Journal of Advanced Manufacturing Technology, 49(1-4), pp. 239251.

[13] Samper, S., Adragna, P.-A., Favreliere, H., and Pillet, M., 2009. "Modeling of 2d and 3d assemblies taking into account form errors of plane surfaces". Journal of Computing and Information Science in Engineering, 9(4), November, p. 041005.

[14] Formosa, F., and Samper, S., 2007. "Modal expression of form defects". In Models for Computer Aided Tolerancing in Design and Manufacturing, J. Davidson, ed. Springer Netherlands, pp. 13-22.

[15] Stoll, T., Wittmann, S., Helwig, S., and Paetzold, K., 2007. "Registration of measured and simulated nonideal geometry using optimization methods". In Proceedings of the 10th CIRP International Seminar on Computer Aided Tolerancing, Erlangen, A. Weckenmann, ed.

[16] Stoll, T., Wittmann, S., and Meerkamm, H., 2009. “Tolerance analysis with detailed part modeling including shape deviations". In Proceedings of the 11th CIRP International Conference on Computer Aided Tolerancing, Annecy, M. Giordano, F. Villeneuve, and L. Mathieu, eds.

[17] Besl, P., and McKay, N. D., 1992. "A method for registration of 3-d shapes". Pattern Analysis and Machine Intelligence, IEEE Transactions on, 14(2), pp. 239256.

[18] Kockara, S., Halic, T., Iqbal, K., Bayrak, C., and Rowe, R., 2007. "Collision detection: A survey". In IEEE International Conference on Systems, Man and Cybernetics, Montreal, pp. 4046-4051.

[19] Zhang, M., Anwer, N., Mathieu, L., and Zhao, H. B., 2011. "A discrete geometry framework for geometrical product specifications". In Proceedings of the $21 \mathrm{st}$ CIRP Design Conference, Kaist, M. K. Thompson, ed.

[20] Zhang, M., Anwer, N., Stockinger, A., Mathieu, L., and Wartzack, S., 2013. "Discrete shape modeling for skin model representation". Proceedings of the Institution of Mechanical Engineers, Part B: Journal of Engineering Manufacture, 227(5), pp. 672-680.

[21] Pottmann, H., Leopoldseder, S., and Hofer, M., 2004. "Registration without icp". Computer Vision and Image Understanding, 95(1), July, pp. 54-71.

[22] Flöry, S., 2009. "Fitting curves and surfaces to point clouds in the presence of obstacles". Computer Aided Geometric Design, 26(2), pp. 192 - 202. 